\title{
Cell-Free DNA: A Novel Biomarker for Patients with Prostate Cancer?
}

\author{
Jörg Ellinger ${ }^{1, *}$ and Patrick J. Bastian ${ }^{2}$
}

\author{
${ }^{1}$ Klinik und Poliklinik für Urologie, Universitätsklinikum Bonn, Rheinische Friedrich-Wilhelms-Universität Bonn, \\ Bonn, Germany \\ ${ }^{2}$ Urologische Klinik und Poliklinik, Universitätsklinikum Großhadern, Ludwig-Maximilians-Universität München, \\ Munich, Germany
}

\begin{abstract}
The presence of small amounts of circulating DNA in plasma was demonstrated 60 years ago. Since then, cell-free DNA has been tested for quantity, fragmentation pattern and tumor-specific sequences in patients with various malignancies. The introduction of improved detection methods showed that all these alterations are regularly detectable in many cancer patients and the investigation of cell-free DNA may provide useful diagnostic and prognostic information. Herein, we review the recent findings on cell-free DNA alterations in patients with prostate cancer and discuss its diagnostic and prognostic potential.
\end{abstract}

Keywords: Cell-free DNA, prostate cancer, biomarker, serum, methylation.

\section{INTRODUCTION}

The presence of circulating cell-free DNA in human plasma was reported in 1948 by Mendel and Metais [1]. The development of improved detection methods led to the discovery of increased circulating DNA levels in cancer patients compared to healthy individuals [2]. It was later confirmed that cancer patients' DNA levels are not only increased compared to healthy individuals, but also to patients with various non-malignant diseases [3]. Finally, it was shown that the circulating DNA carried tumor-specific alterations [4]. The amount of circulating DNA is within the range of nanograms, and thus the detection of circulating DNA was laborious until the development of the polymerase chain reaction (PCR). After development of PCR and quantitative PCR techniques, knowledge of cell-free circulating DNA was rapidly growing: It was shown for many tumor entities that cell-free circulating DNA levels are increased and allow distinguishing patients with non-malignant disease from healthy individuals (e.g. lung [5], colon [6], cervical [7], ovarian [8], breast [9], testis [10], bladder [11] and prostate cancer [12]). Cell-free DNA may therefore serve as a non-invasive universal cancer biomarker.

Despite the 60 years of research, the origin of these DNA fragments in cancer patients remains largely unknown. While the tumor contributes to the circulating DNA, the vast majority originates from healthy cells $[13,14]$. Jahr, et al. further showed that neither endothelial nor tumor-infiltrating T-cells are causative for the increase of cell-free DNA [13]. The induction of apoptosis (anti-CD95 antibody) or liver necrosis (acetaminophen) in a mice model resulted in a distinct increase of cell-free DNA with either high-molecular

*Address correspondence to this author at the_Klinik und Poliklinik für Urologie, Universitätsklinikum Bonn, Sigmund-Freud-Strasse 25, 53105 Bonn, Germany; Tel: +49 2282871 5109; Fax: +49 2282871 1188;

E-mail: joerg.ellinger@ukb.uni-bonn.de
DNA (necrosis) or short, mono- and di-nucleosomal DNA fragments (apoptosis) [13]. Cancer patients' DNA shows an apoptotic as well as a necrotic pattern $[13,15]$, and the pattern seems to be different in various cancer entities: DNA integrity was increased in patients with colon [6], testicular [10], head and neck [16], breast [17] cancer patients indicating predominantly necrotic breakdown. On the other hand, mainly apoptotic DNA fragments were detected in patients with prostate [18] and bladder [11] cancer. Interestingly, tumor-specific sequences were more frequently observed in low-molecular weight than in high-molecular cell-free DNA of prostate cancer patients [19]. The clearance of cell-free DNA from the bloodstream occurs rapidly: fetal DNA disappeared from the blood of mothers after delivery with a halflife time of 16.3 minutes [20]. It is known that cell-free DNA is sensitive to plasma nucleases (e.g. DNase 1), but renal [21] and hepatic [22] clearance are also involved in the elimination of cell-free DNA.

So far, it is unknown if the release of cell-free DNA has any biological effects. Cultured cells have been shown to release double stranded DNA into the media [23], and cellfree DNA might be incorporated into cells [24]. These findings led to the introduction of the concept of "genometastasis" which postulates a horizontal transfer of tumor DNA with transforming potential into stem cells in distant organs [25]. However, this hypothesis remains to be proven.

Circulating DNA can be isolated from both plasma and serum, but serum contains an approximately 6-times higher DNA concentration. It was debated for a long time whether these higher levels are due to a contamination by leukocytes. Recently, Umetani, et al. showed that less than $10 \%$ of the 6fold higher serum DNA levels were due to contamination by other sources (i.e. release from leucocytes during the separation of serum) [26]. The reason for higher serum levels remains unknown; but a loss of DNA in plasma during purification procedures was excluded [26]. Higher DNA levels 
favor the use of serum over plasma, but it is not as simple as that: delayed processing (i.e. $>6$ hours) of blood samples resulted in significantly increased serum but not in plasma DNA concentration [27]. In addition, the size of cell-free DNA increased if the blood was stored longer than 6 hours prior centrifugation, thereby indicating contamination by leukocyte DNA during storage [28]. Thus, the analysis of cell-free DNA requires highly standardized processing procedures.

Cell-free circulating DNA harbors the potential of a useful cancer biomarker. DNA levels, fragmentation patterns and tumor-specific alterations offer interesting possibilities for diagnostic and prognostic purposes. We will discuss the recent findings on cell-free DNA in prostate cancer (PCA) patients with regard to the diagnostic and prognostic information.

\section{DNA LEVELS}

So far, cell-free DNA levels have been assessed in patients with prostate cancer in ten studies [12, 18, 29-36]. Pooled data from several studies including more than 650 PCA patients and 350 control subjects suggest that cell-free
DNA levels provide helpful diagnostic (Table 1) and prognostic (Table 2) information. However, the evaluation of the performance of cell-free DNA as diagnostic/prognostic marker is difficult because different DNA isolation procedures and detection methods have been applied.

Jung, et al. published the first study on cell-free DNA levels in patients with prostate cancer [12]. DNA levels were similar in patients with benign prostate hyperplasia $(\mathrm{BPH})$ and clinically localized PCA, but patients with metastatic PCA had significantly increased cell-free DNA levels. Maybe the use of the less sensitive fluorometric assay is the reason for missing differences between BPH and localized PCA.

Recent studies employed a more sensitive real-time PCR to compare the concentration of cell-free DNA in PCA patients and controls [18, 29-31, 33]. The largest study so far (localized PCA $n=168, B P H n=42$, healthy controls $n=11$ ) was published by Ellinger, et al. [18]. The detection of cellfree DNA provided a sensitivity of $88 \%$ and a specificity of $64 \%$ (area under curve 0.824). A somewhat better discrimination was observed by Altimari, et al. [33] with a sensitivity of $80 \%$ and a specificity of $82 \%$ (localized PCA $n=64, \mathrm{BPH}$

Table 1. DNA Levels: Diagnostic Value

\begin{tabular}{|c|c|c|c|c|c|c|c|}
\hline Study & & n PCA) & n (Control) & Sensitivity & Specificity & AUC & Method \\
\hline \hline Jung 2004 & {$[12]$} & $91 \dagger$ & 93 & n.s. & n.s. & n.s. & fluorometric assay \\
\hline Allen 2004 & {$[29]$} & $27 \ddagger$ & 10 & $85 \%$ & $73 \%$ & n.r. & PCR \\
\hline Papadopoulou 2004 & {$[30]$} & 12 & 18 & $58 \%$ & $94 \%$ & 0.840 & dipstick \\
\hline Papadopoulou 2004 & {$[30]$} & 12 & 13 & $58 \%$ & $92 \%$ & 0.708 & PCR \\
\hline Boddy 2005 & {$[31]$} & 78 & 99 & n.s. & n.s. & n.s. & PCR \\
\hline Chun 2006 & {$[32]$} & 142 & 19 & n.r. & n.r. & n.r. & spectrophotometry \\
\hline Ellinger 2008 & {$[18]$} & 168 & 42 & $88 \%$ & $64 \%$ & 0.824 & PCR \\
\hline Altimari 2008 & {$[33]$} & 64 & 45 & $80 \%$ & $82 \%$ & 0.881 & PCR \\
\hline Cherepanova 2008 & {$[35]$} & 5 & 59 & n.r. & n.r. & n.r. & fluorometric assay \\
\hline Schwarzenbach 2009 & {$[36]$} & 69 & 10 & n.r. & n.r. & n.r. & spectrophotometry \\
\hline
\end{tabular}

Abbreviations: AUC, area under curve; n.s. not significant; n.r., not reported

Note: $\dagger$ mixed cohort of patients with metastatic and localized disease; $\ddagger$ including 12 patients with high grade PIN.

Table 2. DNA Levels: Prognostic Value

\begin{tabular}{|c|c|c|c|c|}
\hline Study & & n (PCA) & Observation & Method \\
\hline \hline Jung 2004 & {$[12]$} & $91 \dagger$ & predictor of cancer specific survival, increased in metastatic PCA & fluorometric assay \\
\hline Bastian 2007 & {$[34]$} & 192 & $\begin{array}{c}\text { predictor of PSA recurrence, correlated with Gleason Score, } \\
\text { surgical margin status and extraprostatic extension }\end{array}$ & PCR \\
\hline Ellinger 2008 & {$[18]$} & 168 & predictor of PSA recurrence & PCR \\
\hline Altimari 2008 & {$[33]$} & 64 & correlated with pT-stage & PCR \\
\hline
\end{tabular}

Abbreviations: AUC, area under curve; n.s. not significant; n.r., not reported

Note: $\uparrow$ mixed cohort of patients with metastatic and localized disease. 
$\mathrm{n}=45$; area under curve 0.881 ). Both studies reported approximately 3-fold increased DNA levels in PCA patients. The smaller series published by Allen et al. $(\mathrm{n}=15)$ and $\mathrm{Pa}-$ padopoulou, et al. $(\mathrm{n}=12)$ showed similar results [29, 30]. Interestingly, Papadopoulou, et al. also reported that a semiquantitative dipstick method (Invitrogen DNA DipStick), which may be less cost-intensive and more rapidly to accomplish, provided similar diagnostic information as a realtime PCR-assay. Using spectrophotometry Chun, et al. also found increased DNA levels in PCA patients, and the predictive value of a multivariate model (including total PSA, free/total PSA, cell-free DNA) was significantly improved by $5.6 \%$ to $78.3 \%$ [32].

In contrast to the above findings, Boddy, et al. failed to detect significant differences between patients with PCA and non-malignant prostate disease [31]. This could be due to the fact that PCA was not definitively excluded at the time of blood withdrawal by histological examination, and thus elevated levels may be due to undetected PCA: Ellinger, et al. also reported increased levels of cell-free DNA in patients with incidental PCA (See Table 1 for details) [18].

In addition to the diagnostic information, cell-free DNA levels may also help to identify PCA patients with poor prognosis. Altimari, et al. showed significant correlation with pathological stage [33]. It was also recently shown that plasma DNA levels of patients with metastatic disease were higher than in patients with localized PCA [36]. Bastian, et al. [34] and Ellinger, et al. [18] demonstrated that cell-free DNA levels were significant predictors of PSA recurrence following radical prostatectomy. Jung, et al. showed that cell-free DNA levels were a predictor of PCA specific survival in patients with metastatic disease (See Table 2 for details) [12].

\section{DNA FRAGMENTATION}

The fragmentation pattern allows conclusions about the underlying cell-death entity of the circulating DNA. Hanley, et al. combined a hybridization-capture technique with a PCR to measure ten different sized plasma DNA fragments (200bp to $10 \mathrm{~kb}$ ) and to calculate the DNA integrity. The DNA integrity was increased in PCA patients $(n=123)$ compared to controls $(n=67$; sensitivity $70 \%$, specificity $68 \%$, area under curve 0.788) [37]. Two other studies employed a quantitative real-time PCR to amplify two different sized PCR products (124bp and 271bp [18], 105bp and 356bp [38]) to assess the fragmentation pattern. Ellinger, et al. reported shorter DNA fragments in patients with PCA $(n=168)$ than in patients with BPH $(n=42$; specificity $81 \%$, sensitivity of $68 \%$, area under curve 0.786 ). Furthermore, the presence of short DNA fragments was a predictor of PSA recurrence following radical prostatectomy [18]. In contrast, Boddy, et al. did not observe a different cell-free DNA fragmentation pattern in PCA $(n=61)$ and BPH patients $(n=62)$ or a correlation with clinical-pathological parameters [38].

The somewhat confusing results may be explained by the use of plasma [37, 38] vs. serum [18] and the different detection methods. However, the studies by Hanley, et al. and Ellinger, et al. suggest a diagnostic potential and future studies are necessary to clarify the role of DNA fragmentation in patients with PCA.

\section{MITOCHONDRIAL DNA}

Two studies investigated plasma/serum cell-free mitochondrial DNA. Interestingly, the concentration of genomic and mitochondrial DNA were not correlated to each other, maybe due to different compartmentalization and degradability of mitochondrial and genomic DNA [39]. Mehra, et al. reported a 3-fold increase of mitochondrial DNA in patients with metastatic PCA compared to controls [39], whereas Ellinger, et al. did not observe differences between patients with clinically localized PCA and BPH [40]. However, mitochondrial DNA levels were an independent predictor of PSA recurrence following radical prostatectomy for clinically localized PCA [40] and high levels of mitochondrial DNA were correlated with PCA-specific survival in patients with advanced PCA [39]. In summary, a diagnostic potential is questionable, but there is a potential role as prognostic biomarker.

\section{TUMOR-SPECIFIC ALTERATIONS}

Tumor-specific sequences in serum/plasma cell-free DNA were first reported in 1989 [4]. Recent studies also confirmed that prostate cancer patients' cell-free DNA includes tumor-specific DNA [19, 30, 33, 34, 41-51]. The amount of tumor-specific DNA is usually limited to a fraction of less than $10 \%,[18,52,53])$. An increase of cell-free DNA seems to be a universal feature of neoplasia, and thus the detection of PCA-specific alterations may be helpful in order to distinguish PCA patients from patients with other malignancies. Recent studies in PCA patients focused on either the detection of epigenetic (DNA hypermethylation) or genetic DNA alterations (allelic imbalances).

\section{DNA Hypermethylation}

DNA hypermethylation is one of the most common and earliest alterations during prostate carcinogenesis. For instance, DNA hypermethylation of the GSTP1 promoter was observed in approximately $90 \%$ of PCA tissues [54]. The detection of aberrantly methylated cytosine is feasible following bisulphite treatment, which induces conversion of unmethylated cytosine to uracil, whereas methylated cytosine remain unchanged. The sequence differences are then detectable using a PCR ("methylation specific PCR") [55]. However, approximately $90 \%$ of the DNA is lost during bisulphite treatment, and thereby limiting the sensitivity [56]. A "methylation sensitive PCR" may be more sensitive: methylation-sensitive restriction enzymes cleave unmethylated DNA, and methylated DNA sequences are thereafter detected using PCR [52].

A number of studies have assessed the presence of GSTP1 hypermethylation in cell-free circulating DNA [18, $30,33,43-45,49-53]$. The sensitivity of these studies ranged from $11-100 \%$ and the specificity was $>93 \%$; the frequency of GSTP1 hypermethylation was $30 \%$ in the pooled analysis of approximately 900 PCA. A multigene analysis increased the sensitivity if three or four gene sites were analysed in combination $[44,49,51,53]$. It was also shown that a DA hypermethylation profile allowed to distinguish tissue of different carcinomas [57]. Our recently published studies on patients with prostate [42], bladder [14] and testicular cancer [58] suggest that a multigene analysis may also be applicable 
in cell-free DNA. See Table 3 for a detailed summary of the diagnostic information of cell-free DNA hypermethylation.

The analysis of cell-free DNA hypermethylation may also provide prognostic information (see Table 4). Bastian, et al. demonstrated that GSTP1 hypermethylation was the strongest predictor of PSA recurrence following radical prostatectomy in patients with clinically localized PCA [52]. Reibenwein, et al reported a correlation between GSTP1 methylation and the Gleason Score respectively the metastatic load in patients with hormone-refractory PCA [44]. Finally, it was recently published that RASSF1A, RARB2 and GSTP1 hypermethylation correlated with the Gleason Score and the serum PSA; RARB2 and GSTP1 methylation also correlated with the AJCC stage [49].

\section{Allelic Imbalances}

So far, five studies have investigated the presence of allelic imbalance (i.e. loss of heterozygosity, LOH; microsatellite instability, MSI). Allelic imbalance requires the comparison of cell-free DNA with patients' leukocyte DNA, and differences are assessed using a PCR. The high background of normal DNA within the circulation limits the sensitivity of the method and is the major drawback. Furthermore, the tests require relatively high amounts of cell-free DNA, and thus the number of informative of analyses varies between 40 and $80 \%$ [48, 49]. Separation into high and lowmolecular DNA during the DNA isolation procedure seems to be an improvement: Muller, et al. demonstrated a higher sensitivity using low- instead of high-molecular DNA for the LOH analyses [19].

Schwarzenbach, et al. were the first to show that the analysis of LOH in cell-free DNA is feasible [46]: However, LOH/MSI was also detected in cell-free DNA of patients with $\mathrm{BPH}[46,47]$. A panel of 13 polymorphic markers provided a sensitivity of $57 \%$ and a specificity of $70 \%$ [47]. Sunami, et al. used a panel of eight markers and observed the presence of at least one $\mathrm{LOH}$ in $47 \%$ of PCA patients, whereas LOH was not observed in healthy controls [49]. A recent study by Schwarzenbach, et al. demonstrated a $45 \%$ sensitivity of AI in cell-free plasma DNA of patients with localized PCA [36]. See Table 5 for a summary of the diag-

Table 3. DNA hypermethylation: Diagnostic value

\begin{tabular}{|c|c|c|c|c|c|c|c|}
\hline Study & & Gene Site & n (PCA) & n (Control) & Sensitivity & Specificity & Method \\
\hline \hline Goess1 2000 & {$[41]$} & GSTP1 & $32 \dagger$ & 22 & $72 \%$ & $100 \%$ & MSP \\
\hline Jeronimo 2002 & {$[43]$} & GSTP1 & 69 & 31 & $36 \%$ & $100 \%$ & MSP \\
\hline Jeronimo 2002 & {$[43]$} & GSTP1 & 69 & 31 & $13 \%$ & $100 \%$ & qMSP \\
\hline Papaopoulou 2004 & {$[30]$} & GSTP1 & $31 \ddagger$ & 9 & $52 \%$ & $100 \%$ & MSP \\
\hline Bastian 2005 & {$[52]$} & GSTP1 & $213 \dagger$ & 35 & $11 \%$ & $100 \%$ & qMS-PCR \\
\hline Reibenwein 2007 & {$[44]$} & GSTP1, AR, 14-3-3-Sigma & $76 \ddagger$ & 49 & $30 \%$ & $100 \%$ & MSP \\
\hline Chuang 2007 & {$[50]$} & GSTP1 & 36 & 27 & $31 \%$ & $93 \%$ & MSP \\
\hline Bryzgunova 2008 & {$[45]$} & GSTP1 & 5 & 10 & $100 \%$ & $100 \%$ & BS \\
\hline Ellinger 2008 & {$[53]$} & GSTP1, TIG1,PTGS2, RPRM & 168 & 42 & $47 \%$ & $93 \%$ & qMS-PCR \\
\hline Altimari 2008 & {$[33]$} & GSTP1 & $18 \dagger$ & 22 & $33 \%$ & $95 \%$ & MSP \\
\hline Bastian 2008 & {$[51]$} & GSTP1, MDR1* & 192 & 35 & $32 \%$ & $100 \%$ & qMS-PCR \\
\hline Sunami 2008 & {$[49]$} & GSTP1, RARB2, RASSF1A & $83 \ddagger$ & 40 & $28 \%$ & $100 \%$ & MSP \\
\hline
\end{tabular}

Abbreviations: MSP, methylation-specific PCR; BS, bisulphite sequencing; qMS-PCR, quantitative methylation-sensitive PCR; qMSP, quantitative methylation-specific PCR Note: $\dagger$ including patients $(<25 \%)$ with metastatic or hormone-refractory prostate cancer; $\ddagger$ including patients $(>60 \%)$ with metastatic or hormone-refractory prostate cancer; $*$ EDNRB, CD44, NEP, PTGS2, RASSF1A, RARB and ESR1 were unmethylated in patients with localized PCA

Table 4. DNA Hypermethylation: Prognostic Value

\begin{tabular}{|c|c|c|c|c|}
\hline Study & & n (PCA) & Observation \\
\hline \hline Bastian 2005 & {$[52]$} & 192 & GSTP1 was a predictor of PSA recurrence \\
\hline Reibenwein 2007 & {$[44]$} & $76 \ddagger$ & RASSF1A, RARB2, GSTP1 correlated with Gleason Score and PSA; \\
RARB2, GSTP1 correlated with AJCC stage
\end{tabular}

Abbreviations: MSP, methylation-specific PCR; qMS-PCR, quantitative methylation-sensitive PCR

Note: $\ddagger$ including patients $(\geq 70 \%)$ with metastatic or hormone-refractory prostate. 
Table 5. Allelic Imbalance: Diagnostic Value

\begin{tabular}{|c|c|c|c|c|c|c|}
\hline Study & & Microsatellite Marker & $\mathbf{n}(\mathbf{P C A})$ & n (Control) & Sensitivity & Specificity \\
\hline Muller 2006 & [46] & $\begin{array}{c}\text { D3S3703, THRB, D6S474, D6S1631, D7S522, D8S87, } \\
\text { D8S137, D8S286, D8S360, D9S171, D9S1748, } \\
\text { D10S1765, D11S898, D11S1313, TP53.6 }\end{array}$ & 65 & 36 & $34 \%$ & $78 \%$ \\
\hline Schwarzenbach 2008 & [47] & $\begin{array}{c}\text { THRB, D6S474, D61631, D7S522, D8S87, D8S137, } \\
\text { D8S286, D8S360, D9S171, D9S1748, } \\
\text { D10S1765, D11S898, D11S1313 }\end{array}$ & 230 & 43 & $57 \%$ & $70 \%$ \\
\hline Sunami 2008 & [49] & D6S286, D8S261, D8S262, D9S171, D10S591, D18S70 & $83 \ddagger$ & 40 & $47 \%$ & $100 \%$ \\
\hline Schwarzenbach 2009 & [36] & $\begin{array}{c}\text { D6S474, D6S1631, D7522, D8S87, D8S137, D8S286, } \\
\text { D8S360, D9S171, D9S1748, D10S1765, D10S541, } \\
\text { D11S898, D11S1313, D17S1855 }\end{array}$ & 69 & 10 & $45 \%$ & $100 \%$ \\
\hline
\end{tabular}

Note: $\ddagger$ including $58(70 \%)$ patients with metastatic prostate cancer.

nostic potential of cell-free DNA. Furthermore, the AI frequency was higher in patients with metastatic disease [36], and genetic alterations also indicated a higher Gleason Score [48].

\section{CONCLUSIONS}

Circulating DNA is a promising non-invasive biomarker in the diagnosis and prognosis of PCA. The detection of tumor-specific alterations as well as DNA fragmentation patterns may further increase the diagnostic information obtained by pure analysis of DNA quantities. However, the evaluation of cell-free DNA needs meticulous standardization, and prospective studies are necessary to confirm the clinical value of circulating DNA fragments in PCA.

\section{ACKNOWLEDGEMENTS}

The work in our lab is supported by grants from the North-Rhine Westphalian Association of Urology (Jörg Ellinger) and the Reinhard-Nagel foundation (Patrick J. Bastian). We thank Dr. Nadja Ellinger for helpful discussions on the manuscript.

\section{REFERENCES}

[1] Mandel P, Metais P. Les acides nucleiques du plasma sanguine chez l'homme. C R Acad Sci Paris 1948; 142: 241-3.

[2] Leon SA, Shapiro B, Sklaroff DM, Yaros MJ. Free DNA in the serum of cancer patients and the effect of therapy. Cancer Res 1977; 37: 646-50.

[3] Shapiro B, Chakrabarty M, Cohn EM, Leon SA. Determination of circulating DNA levels in patients with benign or malignant gastrointestinal disease. Cancer 1983; 51: 2116-20.

[4] Stroun M, Anker P, Maurice P, et al. Neoplastic characteristics of the DNA found in the plasma of cancer patients. Oncology 1989; 46: 318-22.

[5] Sozzi G, Conte D, Mariani L, et al. Analysis of circulating tumor DNA in plasma at diagnosis and during follow-up of lung cancer patients. Cancer Res 2001; 61: 4675-8.

[6] Umetani N, Kim J, Hiramatsu S, et al. Increased integrity of free circulating DNA in sera of patients with colorectal or periampullary cancer: direct quantitative PCR for ALU repeats. Clin Chem 2006; 52: 1062-9.

[7] Trejo-Becerril C, Perez-Cardenas E, Trevino-Cuevas H, et al. Circulating nucleosomes and response to chemotherapy: an in vitro, in vivo and clinical study on cervical cancer patients. Int $\mathrm{J}$ Cancer 2003; 104: 663-8.
[8] Chang HW, Lee SM, Goodman SN, et al. Assessment of plasma DNA levels, allelic imbalance, and CA 125 as diagnostic tests for cancer. J Natl Cancer Inst 2002; 94: 1697-703.

[9] Huang ZH, Li LH, Hua D. Quantitative analysis of plasma circulating DNA at diagnosis and during follow-up of breast cancer patients. Cancer Lett 2006; 243: 64-70.

[10] Ellinger J, Wittkamp V, Albers P, et al. Cell-free circulating DNA: diagnostic value in patients with testicular germ cell cancer. J Urol 2009; 181: 363-71.

[11] Ellinger J, Bastian PJ, Ellinger N, et al. Apoptotic DNA fragments in serum of patients with muscle invasive bladder cancer: a prognostic entity. Cancer Lett 2008; 264: 274-80.

[12] Jung K, Stephan C, Lewandowski M, et al. Increased cell-free DNA in plasma of patients with metastatic spread in prostate cancer. Cancer Lett 2004; 205: 173-80.

[13] Jahr S, Hentze H, Englisch S, et al. DNA fragments in the blood plasma of cancer patients: quantitations and evidence for their origin from apoptotic and necrotic cells. Cancer Res 2001; 61: 1659-65.

[14] Ellinger J, El Kassem N, Heukamp LC, et al. Hypermethylation of cell-free serum DNA indicates worse outcome in patients with bladder cancer. J Urol 2008; 179: 346-52.

[15] Wu TL, Zhang D, Chia JH, et al. Cell-free DNA: measurement in various carcinomas and establishment of normal reference range. Clin Chim Acta 2002; 321: 77-87.

[16] Wang BG, Huang HY, Chen YC, et al. Increased plasma DNA integrity in cancer patients. Cancer Res 2003; 63: 3966-8.

[17] Umetani N, Giuliano AE, Hiramatsu SH, et al. Prediction of breast tumor progression by integrity of free circulating DNA in serum. J Clin Oncol 2006; 24: 4270-6.

[18] Ellinger J, Bastian PJ, Haan KI, et al. Noncancerous PTGS2 DNA fragments of apoptotic origin in sera of prostate cancer patients qualify as diagnostic and prognostic indicators. Int J Cancer 2008; 122: $138-43$.

[19] Muller I, Beeger C, Alix-Panabieres C, et al. Identification of loss of heterozygosity on circulating free DNA in peripheral blood of prostate cancer patients: potential and technical improvements. Clin Chem 2008; 54: 688-96.

[20] Lo YM, Zhang J, Leung TN, et al. Rapid clearance of fetal DNA from maternal plasma. Am J Hum Genet 1999; 64: 218-24.

[21] Botezatu I, Serdyuk O, Potapova G, et al. Genetic analysis of DNA excreted in urine: a new approach for detecting specific genomic DNA sequences from cells dying in an organism. Clin Chem 2000; 46: $1078-84$

[22] Minchin RF, Carpenter D, Orr RJ. Polyinosinic acid and polycationic liposomes attenuate the hepatic clearance of circulating plasmid DNA. J Pharmacol Exp Ther 2001; 296: 1006-12.

[23] Anker P, Stroun M, Maurice PA. Spontaneous release of DNA by human blood lymphocytes as shown in an in vitro system. Cancer Res 1975; 35: 2375-82.

[24] Bergsmedh A, Szeles A, Henriksson M, et al. Horizontal transfer of oncogenes by uptake of apoptotic bodies. Proc Natl Acad Sci USA 2001; 98: 6407-11. 
[25] Garcia-Olmo DC, Ruiz-Piqueras R, Garcia-Olmo D. Circulating nucleic acids in plasma and serum (CNAPS) and its relation to stem cells and cancer metastasis: state of the issue. Histol Histopathol 2004; 19: 575-83.

[26] Umetani N, Hiramatsu S, Hoon DS. Higher amount of free circulating DNA in serum than in plasma is not mainly caused by contaminated extraneous DNA during separation. Ann N Y Acad Sci 2006; 1075: 299-307.

[27] Tsui NB, Ng EK, Lo YM. Stability of endogenous and added RNA in blood specimens, serum, and plasma. Clin Chem 2002; 48: 1647-53.

[28] Chan KC, Yeung SW, Lui WB, Rainer TH, Lo YM. Effects of preanalytical factors on the molecular size of cell-free DNA in blood. Clin Chem 2005; 51: 781-4.

[29] Allen D, Butt A, Cahill D, et al. Role of cell-free plasma DNA as a diagnostic marker for prostate cancer. Ann N Y Acad Sci 2004; 1022: 76-80.

[30] Papadopoulou E, Davilas E, Sotiriou V, et al. Cell-free DNA and RNA in plasma as a new molecular marker for prostate cancer. Oncol Res 2004; 14: 439-45.

[31] Boddy JL, Gal S, Malone PR, Harris AL, Wainscoat JS. Prospective study of quantitation of plasma DNA levels in the diagnosis of malignant versus benign prostate disease. Clin Cancer Res 2005; 11: 1394-9.

[32] Chun FK, Muller I, Lange I, et al. Circulating tumour-associated plasma DNA represents an independent and informative predictor of prostate cancer. BJU Int 2006; 98: 544-8.

[33] Altimari A, Grigioni AD, Benedettini E, et al. Diagnostic role of circulating free plasma DNA detection in patients with localized prostate cancer. Am J Clin Pathol 2008; 129: 756-62.

[34] Bastian PJ, Palapattu GS, Yegnasubramanian S, et al. Prognostic value of preoperative serum cell-free circulating DNA in men with prostate cancer undergoing radical prostatectomy. Clin Cancer Res 2007; 13: 5361-7.

[35] Cherepanova AV, Tamkovich SN, Bryzgunova OE, Vlassov VV, Laktionov PP. Deoxyribonuclease activity and circulating DNA concentration in blood plasma of patients with prostate tumors. Ann N Y Acad Sci 2008; 1137: 218-21.

[36] Schwarzenbach H, Alix-Panabieres C, Muller I, et al. Cell-free tumor DNA in blood plasma as a marker for circulating tumor cells in prostate cancer. Clin Cancer Res 2009; 15: 1032-8.

[37] Hanley R, Rieger-Christ KM, Canes D, et al. DNA integrity assay: a plasma-based screening tool for the detection of prostate cancer. Clin Cancer Res 2006; 12: 4569-74.

[38] Boddy JL, Gal S, Malone PR, et al. The role of cell-free DNA size distribution in the management of prostate cancer. Oncol Res 2006; 16: 35-41.

[39] Mehra N, Penning M, Maas J, et al. Circulating mitochondrial nucleic acids have prognostic value for survival in patients with advanced prostate cancer. Clin Cancer Res 2007; 13: 421-6.

[40] Ellinger J, Mueller SC, Wernert N, von Ruecker A, Bastian PJ. Mitochondrial DNA in serum of patients with prostate cancer: a predictor of biochemical recurrence following prostatectomy. BJU Int 2008; 102: 628-32.

[41] Goessl C, Krause H, Muller M, et al. Fluorescent methylationspecific polymerase chain reaction for DNA-based detection of prostate cancer in bodily fluids. Cancer Res 2000; 60: 5941-5.
[42] Ellinger J, Haan K, Heukamp LC, et al. CpG Island hypermethylation in cell-free serum DNA identifies patients with localized prostate cancer. Prostate 2008; 68: 42-9.

[43] Jeronimo C, Usadel H, Henrique R, et al. Quantitative GSTP1 hypermethylation in bodily fluids of patients with prostate cancer. Urology 2002; 60: 1131-5.

[44] Reibenwein J, Pils D, Horak P, et al. Promoter hypermethylation of GSTP1, AR, and 14-3-3sigma in serum of prostate cancer patients and its clinical relevance. Prostate 2007; 67: 427-32.

[45] Bryzgunova OE, Morozkin ES, Yarmoschuk SV, Vlassov VV, Laktionov PP. Methylation-specific sequencing of GSTP1 gene promoter in circulating/extracellular DNA from blood and urine of healthy donors and prostate cancer patients. Ann N Y Acad Sci 2008; 1137: 222-5.

[46] Muller I, Urban K, Pantel K, Schwarzenbach H. Comparison of genetic alterations detected in circulating microsatellite DNA in blood plasma samples of patients with prostate cancer and benign prostatic hyperplasia. Ann NY Acad Sci 2006; 1075: 222-9.

[47] Schwarzenbach H, Chun FK, Muller I, et al. Microsatellite analysis of allelic imbalance in tumour and blood from patients with prostate cancer. BJU Int 2008; 102: 253-8.

[48] Schwarzenbach H, Chun FK, Lange I, et al. Detection of tumorspecific DNA in blood and bone marrow plasma from patients with prostate cancer. Int J Cancer 2007; 120: 1465-71.

[49] Sunami E, Shinozaki M, Higano CS, et al. Multimarker circulating DNA assay for assessing blood of prostate cancer patients. Clin Chem 2009; 55: 559-67.

[50] Chuang CK, Chu DC, Tzou RD, et al. Hypermethylation of the $\mathrm{CpG}$ islands in the promoter region flanking GSTP1 gene is a potential plasma DNA biomarker for detecting prostate carcinoma. Cancer Detect Prev 2007; 31: 59-63.

[51] Bastian PJ, Palapattu GS, Yegnasubramanian S, et al. CpG island hypermethylation profile in the serum of men with clinically localized and hormone refractory metastatic prostate cancer. J Urol 2008; 179: 529-34.

[52] Bastian PJ, Palapattu GS, Lin X, et al. Preoperative serum DNA GSTP1 CpG island hypermethylation and the risk of early prostatespecific antigen recurrence following radical prostatectomy. Clin Cancer Res 2005; 11: 4037-43.

[53] Ellinger J, Bastian PJ, Jurgan T, et al. CpG island hypermethylation at multiple gene sites in diagnosis and prognosis of prostate cancer. Urology 2008; 71: 161-7.

[54] Bastian PJ, Yegnasubramanian S, Palapattu GS, et al. Molecular biomarker in prostate cancer: the role of $\mathrm{CpG}$ island hypermethylation. Eur Urol 2004; 46: 698-708.

[55] Herman JG, Graff JR, Myohanen S, Nelkin BD, Baylin SB. Methylation-specific PCR: a novel PCR assay for methylation status of CpG islands. Proc Natl Acad Sci U S A 1996; 93: 9821-6.

[56] Grunau C, Clark SJ, Rosenthal A. Bisulfite genomic sequencing: systematic investigation of critical experimental parameters. $\mathrm{Nu}$ cleic Acids Res 2001; 29: E65.

[57] Esteller M, Corn PG, Baylin SB, Herman JG. A gene hypermethylation profile of human cancer. Cancer Res 2001; 61: 3225-9.

[58] Ellinger J, Albers P, Perabo FG, et al. CpG Island Hypermethylation of cell-free circulating serum DNA in patients with testicular cancer. J Urol 2009; 182: 324-9. 